\title{
Direct metal-carbon bonding in symmetric bis(C-H) agostic Nickel(I) complexes
}

Weiying He, ${ }^{1,2}$ D. Dawson Beattie, ${ }^{2}$ Hao Zhou, ${ }^{1,2}$ Eric Bowes,${ }^{2}$ Laurel L Schafer, ${ }^{2}$ Jennifer A Love,,$^{1,2}$ and Pierre Kennepohl ${ }^{1,2 *}$

1. Department of Chemistry, University of Calgary, 2500 University Drive NW Calgary Alberta T2N 1N4, Canada.

2. Department of Chemistry, The University of British Columbia, 2036 Main Mall, Vancouver, BC V6T 1Z1, Canada.

pierre.kennepohl@ucalgary

Abstract: Agostic interactions are examples of $\sigma$-type interactions, typically resulting from interactions between $\mathrm{C}-\mathrm{H} \sigma$-bonds with empty transition metal d orbitals. Such interactions often reflect the first step in transition metal-catalysed $\mathrm{C}-\mathrm{H}$ activation processes and thus are of criticial importance in understanding and controlling $\sigma$ bond activation chemistries. Herein, we report on the unusual electronic structure of linear electron-rich $d^{9} \mathrm{Ni}(\mathrm{I})$ complexes with symmetric bis $(\mathrm{C}-\mathrm{H})$ agostic interactions. A combination of $\mathrm{Ni} \mathrm{K}$ edge and $\mathrm{L}$ edge XAS with supporting TD-DFT/DFT calculations reveals an unconventional covalent agostic interaction with limited contributions from the valence $\mathrm{Ni} 3 d$ orbitals. The agostic interaction is driven via the empty $\mathrm{Ni} 4 \mathrm{p}$ orbitals. The surprisingly strong $\mathrm{Ni} 4 \mathrm{p}$-derived agostic interaction is dominated by $\sigma$ contributions with minor $\pi$ contributions. The resulting ligand-metal donation occurs directly along the C-Ni bond axis, reflecting a novel mode of bis- agostic bonding.

\section{Introduction}

Metal-mediated activation and functionalisation of $\mathrm{C}-\mathrm{H}$ bonds is one of the most influential strategies for the efficient synthesis of high-value products and materials. ${ }^{1,2}$ In many instances, $\mathrm{C}-\mathrm{H}$ bond activation proceeds through the initial formation of a direct interaction between the metal ion and the $\mathrm{C}-\mathrm{H}$ functionality. The formation of a $\sigma$ complex, where a $\mathrm{CH}$ moiety acts as a donor towards a metal, is thus often a prerequisite for bond activation and functionalisation. . $^{3,4}$ Intramolecular $\sigma$ interactions of this type are often termed agostic interactions, which are more commonly observed due to the significantly lower entropic penalty for bond formation (i.e., the 
chelate effect $\left.{ }^{5}\right)$. Studies of agostic complexes have dramatically increased our understanding of $\sigma$ complexes even though most untethered $\sigma$ complexes are transient species that are very difficult to trap and investigate. ${ }^{6,7}$

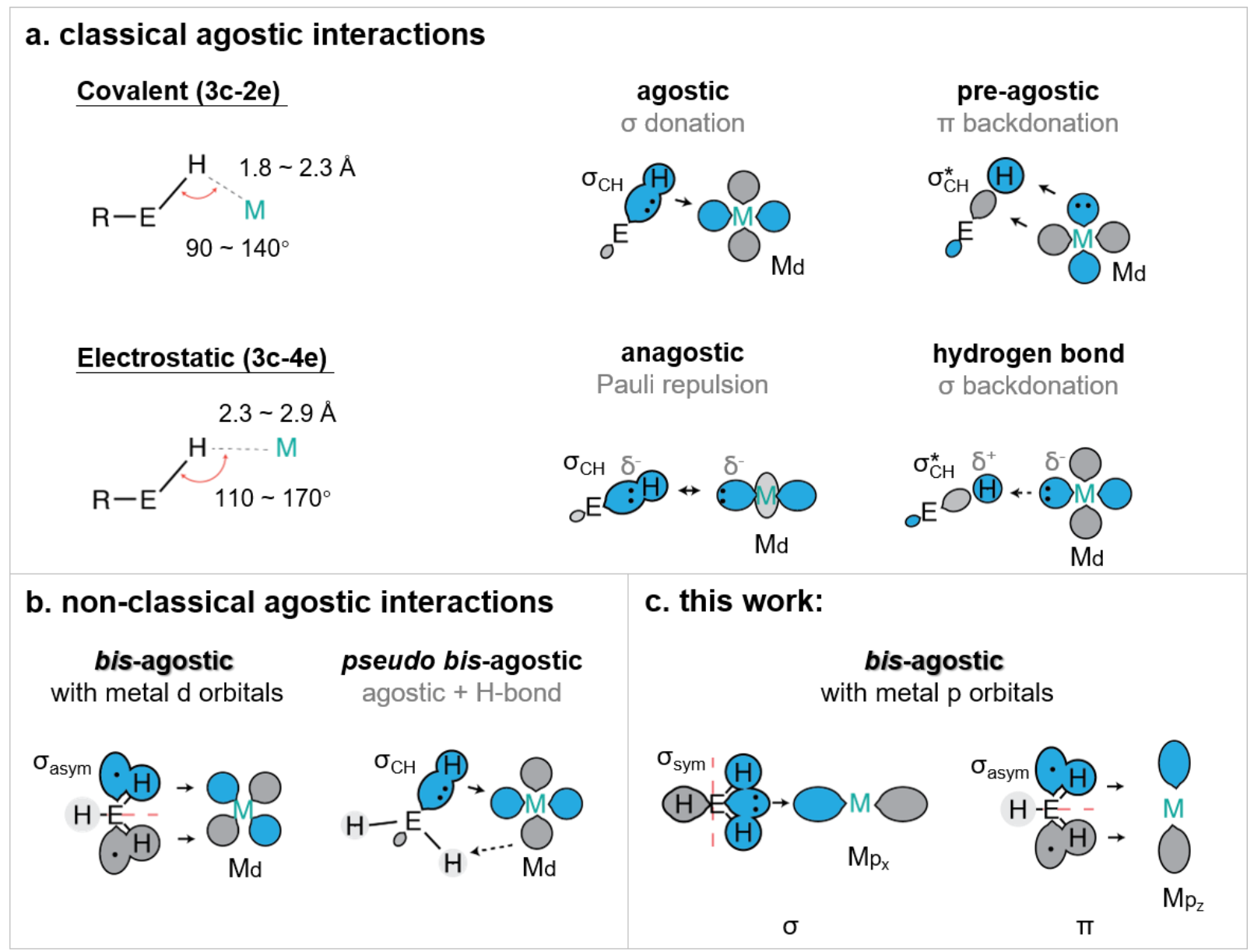

Fig. 1 | a, Geometrical definition of various transition metal - hydrogen interactions: agostic, pre-agostic, anagostic and hydrogen bond $(E=C) ; 8$ b, Summary of orbital interaction scheme for previous bis $\mathrm{M}$ - H-E interaction $(E=C, S i, B)$. c, New p orbital type bis-agostic interaction modes found in this work $(E=C)$.

Our understanding of the fundamental nature of $\mathrm{M}---\mathrm{H}-\mathrm{C}$ interactions continues to evolve, but it has become clear that the degree and directionality of charge redistribution upon bond formation plays a critical role in defining their reactivity. ${ }^{9}$ For example, if the $\mathrm{CH}$ moiety acts as a donor towards an electron-deficient metal centre (acting as a $\sigma$ donor), the interaction is defined as a $\sigma_{\mathrm{CH}}$ interaction. ${ }^{10-13}$ Such a bond is often formally described as $M_{d} \leftarrow \sigma_{\mathrm{CH}}$ donation, reflecting the fact that metal $d$ orbitals act as acceptors. ${ }^{11}$ Charge transfer leads to weakening of the $\sigma_{\mathrm{CH}}$ bond, which is a requisite first step in a wide range of $\sigma$-bond activation methodologies, including ambiphilic ligand-metal activation ${ }^{14} /$ cyclometallation deprotonation $^{15}$ (AMLA/CMD), oxidative addition $(\mathrm{OA}),{ }^{16} \sigma$ bond metathesis (SBM), ${ }^{17}$ and $2+1$ addition. ${ }^{18,19} \mathrm{~A}$ better understanding of different modes of $\sigma$-bond activation may serve to further boost development of $\mathrm{C}-\mathrm{H}$ 
functionalization chemistry. ${ }^{20-23}$ Other modes of $(\mathrm{M}---\mathrm{H}-\mathrm{C})$ binding are also known. For example,electron-rich metal centres may form 3c-4e hydrogen bonds with an electron-deficient $\mathrm{CH}$ moiety. ${ }^{24}$ One must also consider potential anagostic interactions where Pauli repulsion between the metal centre and the $\sigma_{\mathrm{CH}}$ is overcome by steric factors. ${ }^{25,26}$ These different interactions are often classified based on geometric parameters as they have different geometric preferences (Fig. 1a): the $\mathrm{MH}-\mathrm{C}$ angle is more acute for $\sigma$ complexes than for hydrogen bonds. ${ }^{8}$ Such interactions have also been observed with other $\mathrm{E}-\mathrm{H}$ moieties, such as boranes and silanes. ${ }^{27-29}$

Certain agostic complexes include more unusual bonding motifs - these are sometimes termed nonclassical agostic complexes. A prime example is that of "bis-agostic" interactions, where steric constraints enforce a geometry where two adjacent $\mathrm{E}-\mathrm{H}$ bonds are in close proximity to the metal centre (Fig. 1b). Symmetric bis-agostic complexes have been described as resulting from a single $4 \mathrm{c}-2 \mathrm{e}$ interaction between a delocalised $\mathrm{H}-\mathrm{E}-\mathrm{H}(\mathrm{E}=\mathrm{B}, \mathrm{Si}, \mathrm{C})$ fragment orbital and a single empty metal d orbital. ${ }^{30-32}$ Cases of asymmetric binding of the $\mathrm{H}-\mathrm{E}-\mathrm{H}$ moiety (termed pseudo-bis-agostic bonds) often result from a dominant classical agostic interaction and a secondary hydrogen bond. ${ }^{30,33}$ We previously reported the linear nickel(I) complexes with pairs of nearly symmetric $\delta$-bis $(\mathrm{C}-\mathrm{H})$ contacts from pendant methyl groups that were tentatively assigned as pseudo-bis-agostic complexes based on existing norms for the assignment of such species. ${ }^{34,35}$ Herein, we report additional spectroscopic data that correct our original assignment and identify a previously unrecognized mode of bis-agostic interaction that results from direct C$\mathrm{Ni}$ bonding dominated by $\mathrm{Ni} 4 \mathrm{p}$ acceptor character with only minimal $\mathrm{Ni} 3 \mathrm{~d}$ contributions. As a result, we consider these linear $\mathrm{Ni}(\mathrm{I})$ agostic complexes as the first examples of a new class of agostic complexes in organometallic chemistry.

\section{Results and Discussion}

Ni K edge XAS studies. The first indication that these complexes were unusual came from the comparison of the experimental Nickel K-edge X-ray absorption near edge structure (XANES) spectra for complexes 1-4 (Fig. 2a). ${ }^{34,35}$ Fig. 2b shows the Ni K-edge pre-edge region for analogous complexes both with $(\mathbf{1 , 3})$ and without $(2,4)$ bis-agostic interactions: the spectra are strikingly different in this region. The spectra are generally consistent with previously reported $\mathrm{Ni}$ K-edge spectra of $\mathrm{Ni}(\mathrm{I})$ complexes $^{36-38}$ wherein a weak electric-quadrupole allowed $\mathrm{Ni} 3 \mathrm{~d} \leftarrow 1 \mathrm{~s}$ 
transition is observed at $8330-8332 \mathrm{eV}$, in addition to an intense dipole-allowed $\mathrm{Ni} 4 \mathrm{p} \leftarrow 1 \mathrm{~s}$ feature at $28337 \mathrm{eV}$. However, the $\mathrm{Ni} 4 \mathrm{p} \leftarrow 1 \mathrm{~s}$ feature ${ }^{39}$ exhibits large differences depending on the presence/absence of the agostic interaction. The non-agostic complexes 2 and $\mathbf{4}$ exhibit broad overlapping features with three observable components in the second derivative of the data, see insets in Fig. 2b, 2c); these features collapse into two components (for complexes $\mathbf{1}$ and $\mathbf{3}$ ) in the presence of the tethered methyl group adjacent to the metal centre. Based on the 2nd derivatives and relative intensities of these features, the simplest interpretation is that the two higher energy features merge when the agostic interactions are present (Supplementary Fig.3). Such large effects in the $4 p$ features in K-edge spectroscopy have previously been observed in $\mathrm{Cu}(\mathrm{I})$ complexes, where these features are highly sensitive to the coordination environment around the metal centre. ${ }^{40}$

a
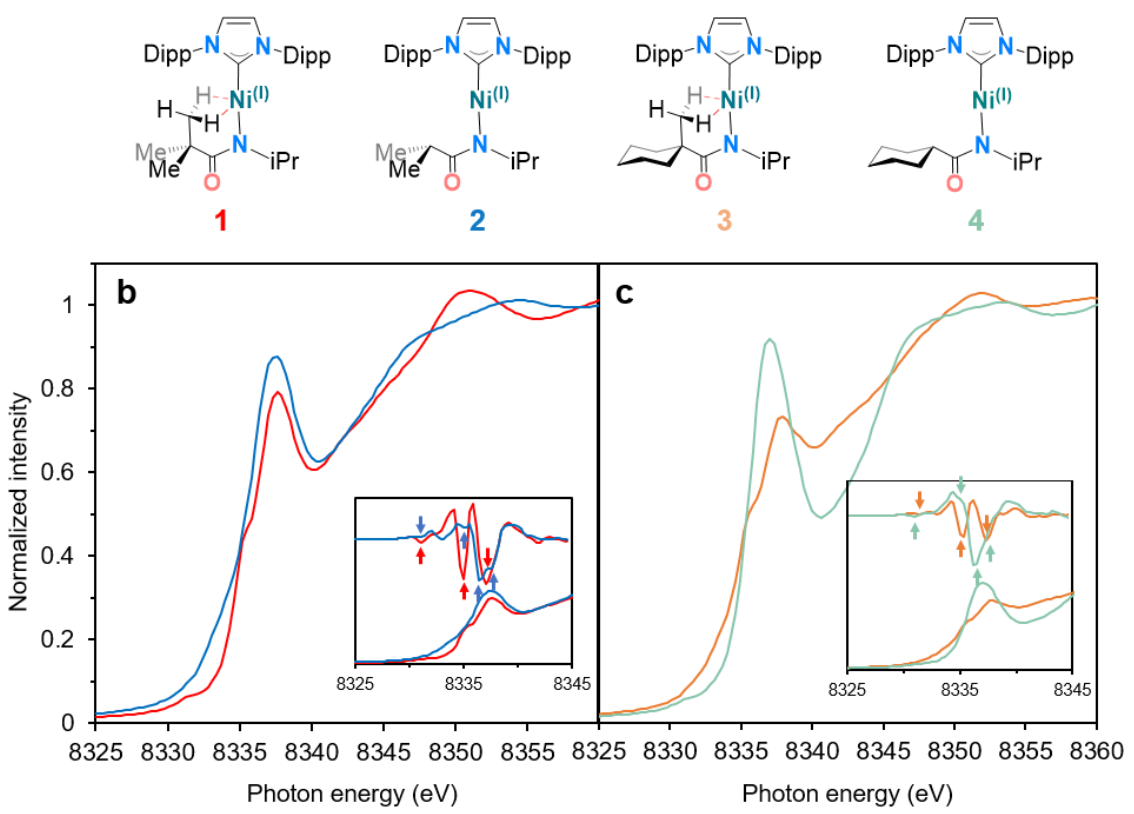

Fig 2 | a, complexes 1-4 studied in this work. b, Normalized Ni K-edge PFY XANES edge spectra for agostic complex (1) and its control group complex non-agostic complexes (2). c, Normalized Ni K-edge PFY XANES edge spectra for agostic complex (3) and its control group complex (4). The pre-edge region of the spectra is shown in the inset with their second derivative spectra used for searching a local maximum of spectrum features, due to the limitation of $\mathrm{Ni} \mathrm{K}$-edge pre-edge resolution. ${ }^{41}$ Potential local maxima of each spectrum are marked with arrow in the same colour of the corresponding spectra.

TD-DFT calculations \& simulation of Ni K edge XAS. To understand the origins of the observed differences in the Ni K-edge spectra, we employed time-dependent density functional theory (TD-DFT) calculations to simulate the experimental XAS spectra. DFT-optimized structures for each of the complexes at the PBE0/Def2-TZVP level of theory are in good agreement with experimental solid-state molecular structures ${ }^{34}$ (Supplementary Table 27). Furthermore, the 
calculated Ni K edge XANES spectra (Fig. 3) reproduce differences observed in the experimental data. The calculated spectra exhibit four distinct features in the pre-edge region (Fig. 3, features A-D). The lowest-lying and weakest feature $(A, \sim 8330 \mathrm{eV})$ corresponds to the electric-dipole forbidden $\mathrm{Ni} 3 \mathrm{~d} \leftarrow 1 \mathrm{~s}$ transition expected for a $3 \mathrm{~d}^{9} \mathrm{Ni}(\mathrm{I})$ metal ion. Any differences in this low energy feature would be difficult to observe given their weak intensity; the feature reflects the electric dipole forbidden excitation of a $\mathrm{Ni}$ 1s core electron into the half-occupied SOMO, which is assigned as the $\mathrm{Ni} 3 \mathrm{~d}_{\mathrm{x} 2-\mathrm{y}_{2}}$ orbital. A more intense energy feature at higher energy $(\mathrm{B}, \sim 8335 \mathrm{eV})$ is assigned as a transition to a ligand-based $\pi_{C N i N}^{*}$ final state that reflects metal-mediated $\pi$ delocalization across the amido and NHC ligands (Supplementary Figure $17 \& 18$ ). This feature remains essentially invariant across the series but gains electric dipole intensity from symmetryallowed $\mathrm{Ni} 3 \mathrm{~d} / 4 \mathrm{p}$ mixing. This $\mathrm{m}$ interaction is responsible for the fixed relative orientation of the two ligands and is perpendicular to the $\mathrm{H}_{3} \mathrm{C}$-Ni axis. Together, the lower energy features $\mathrm{A} \& \mathrm{~B}$ in the Ni K-edge spectra thus reflect the nature of bonding along the primary axis of these linear $\mathrm{Ni}(\mathrm{I})$ complexes; contributions to these features due to agostic interactions are relatively subtle. As anticipated, the agostic interactions do not dramatically influence of inherent nature of the bonding in these metal complexes.

By contrast, agostic contributions are much more significant in the higher energy region of the spectrum. Most notably, the splitting of features $C$ and $D$ decreases substantially in the presence of the agostic methyl group in $\mathbf{1 / 3}$ as compared to $2 / 4$. More specifically, the energy of feature $C$ is much higher in the agostic complexes (by $\sim 0.6 \mathrm{eV}$ ). This feature corresponds to transitions with large $\mathrm{Ni} 4 \mathrm{p}_{\mathrm{x}}$ character; this acceptor orbital is thus strongly affected by the presence of the agostic interaction. Feature $D$, on the other hand, is much less affected by the agostic interaction. Qualitatively, the effect is the same in both pairs of complexes, albeit the decrease in C/D splitting is more pronounced in $\mathbf{1}$ than in $\mathbf{3}$. Simulated spectra based on the TD-DFT calculations reasonably reproduce the experimentally observed pre-edge data using these four main features (see Fig.3 \& Table 1), indicating that differences in the Ni K-edge spectra are well described by the electronic structure calculations. 
a

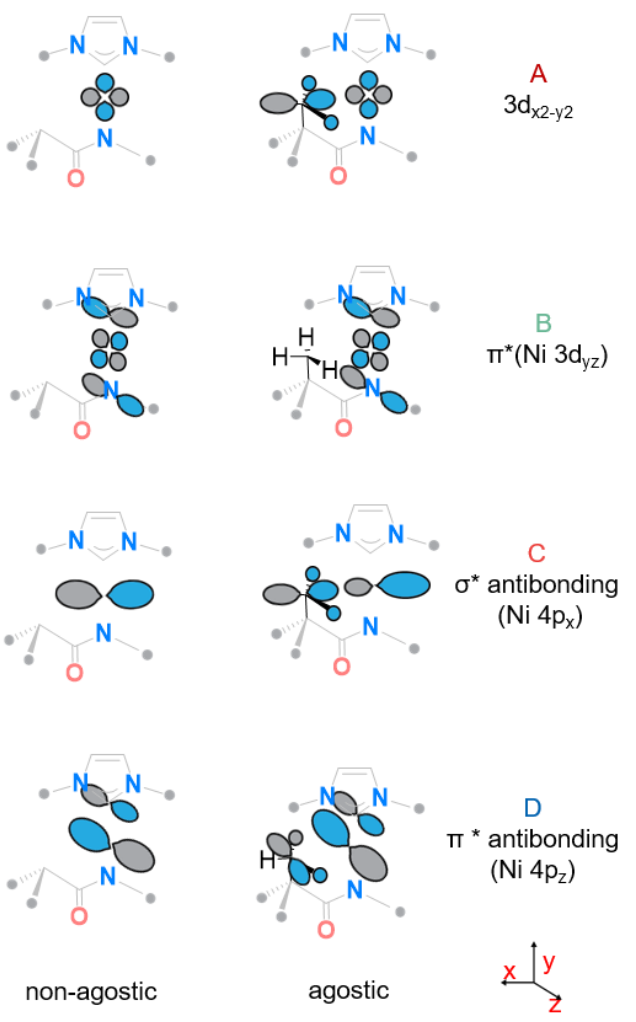

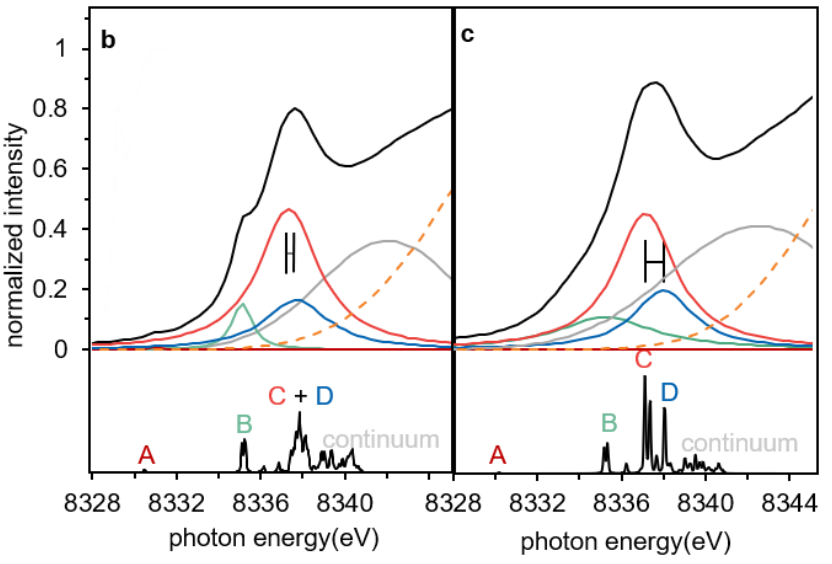

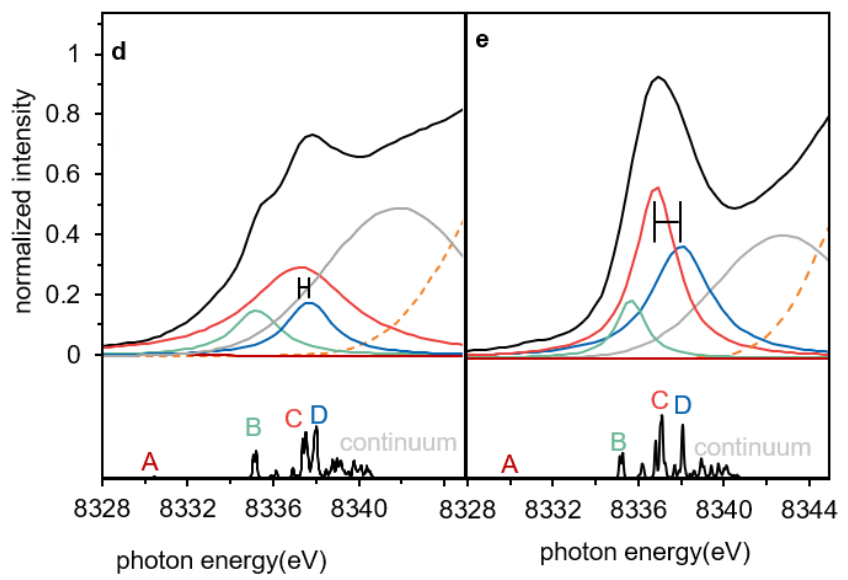

Fig 3 | a, Schematic molecular orbital acceptors contributed to the calculated transition features. b-e, The experimental spectrum simulated with four Lorentzian function curves and one Gaussian curve (top), and the TD-DFT calculated Ni K-edge XAS spectra (bottom) of complexes 1-4, 1 (b), 2 (c), 3 (d), 4 (e). The difference of $4 p_{x} \leftarrow 1 s$ transition (feature $C$ ) and $4 p_{z} \leftarrow 1 s$ transition (feature $D$ ) between agostic complexes and non-agostic complexes in the simulating features are highlighted a horizontal error bar. Four isolated pre-edge features are labelled with $A-D$, each label is highlighted in the same colour as the assigned simulating curve. All the TD-DFT calculated transition energy was shifted by $180.19 \mathrm{eV}$ for a better comparison with experimental data. See details of calculated energy in Supplementary Figure 4-8 and Supplementary Table 1-12 and simulated energy in Supplementary Figure 9-12 and Supplementary Table 13-16.

The observed changes in the $4 p_{x, z}$ final states can be explained, as shown in Figure 4. The $\mathrm{Ni}$ $3 d$ orbitals cannot contribute significantly to interactions with the agostic methyl group. However, the empty $4 p_{x, z}$ orbitals have the correct symmetry to interact with appropriate donor orbitals on methyl group. The agostic methyl group has the correct orientation to interact with the empty $\mathrm{Ni}$ $4 p_{x, z}$ orbitals and thus generate donor-acceptor interactions between the methyl group and the metal centre. The dominant $\sigma$-type interaction reflects a $\left(\mathrm{C}_{2 p}+\mathrm{H}_{1 \mathrm{~s}}\right) \rightarrow \mathrm{Ni}_{4 p x}$ donor interaction which yields an increase in the energy of the $\mathrm{Ni} 4 \mathrm{p}_{\mathrm{x}}$ orbitals by $>0.5 \mathrm{eV}$. The symmetry-allowed $\pi$ interaction is extremely weak, due to both poor overlap and the existing involvement of the $4 p_{z}$ orbital in $\pi$ bonding with the $\mathrm{NHC}$ and amido ligands. 


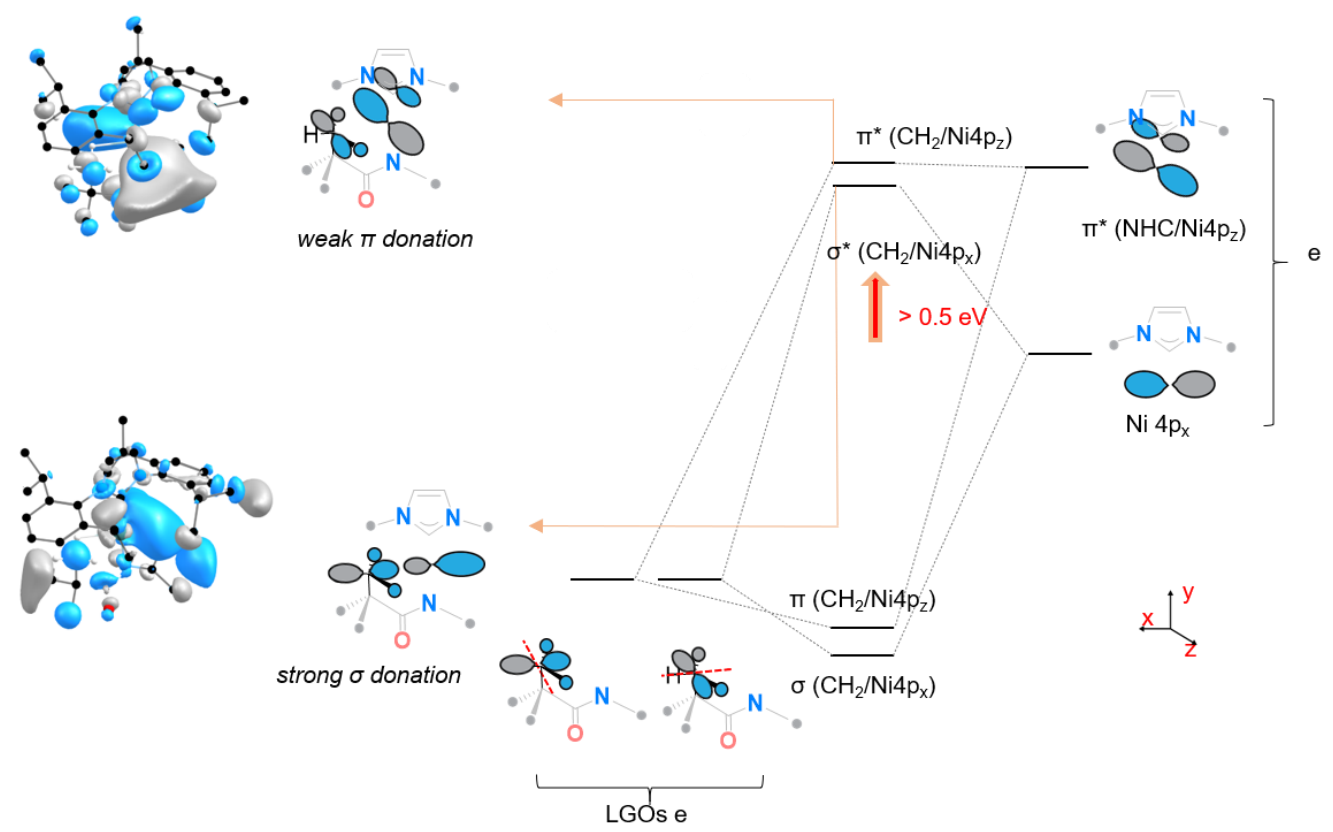

Fig. 4 | Molecular orbital representation of the agostic interaction contributed by the frontier $\mathrm{Ni} 4 \mathrm{p}_{\mathrm{x} / \mathrm{z}}$ orbital and the ligand group orbital of three $\mathrm{C}-\mathrm{H} \sigma$ bonding orbital. The inset antibonding orbitals are representing $\sigma$ donation and $\pi$ donation through agostic interactions. Hydrogen atoms except ones in pendant methyl substitute in the MO graph are omitted for a better view of the frontier orbital interaction.

A more detailed evaluation of the DFT results allows us to estimate the importance and relevance of the agostic interaction in these complexes. The influence of the agostic interaction can be (broadly) estimated via the increase in energy of feature $C$ relative to the non-agostic analogue. From this, we estimate that the orbital contribution to the agostic $\sigma$ donation is surprisingly large in both systems ${ }^{42}$ yet is somewhat larger in $1(\sim 65 \mathrm{~kJ} / \mathrm{mol})$ than in $3(\sim 50 \mathrm{~kJ} / \mathrm{mol})$. These differences are consistent with observed differences in steric repulsion between the two systems. The cyclohexyl substituent exhibits a larger steric clash with the NHC ligand substituent (Fig.5a), which causes a distortion of the $\mathrm{CH}_{2}---\mathrm{Ni}$ agostic interaction (Fig. 5a) and thus weakens the carbon-nickel interaction. To probe the sensitivity of the $\mathrm{Ni} 4 \mathrm{p}_{\mathrm{x}, \mathrm{z}}$ final states to details of the agostic interaction, a relaxed surface scan was performed on 2 (Fig.5b) starting from its ground state geometry and scanning about the $\mathrm{N}-\mathrm{C}-\mathrm{C}-\mathrm{H}$ dihedral angle $\left(\mathrm{d}_{\mathrm{CNNH}}\right)$. Energetically, the formation of the agostic interaction $\left(100^{\circ}<\mathrm{d}_{\mathrm{CNNH}}<120^{\circ}\right)$ is not favourable due to steric repulsion by forcing the methyl group towards the metal centre (Supplementary Figure 20). Electronically, the effect of the agostic interaction has a large impact on the $\mathrm{Ni} 4 \mathrm{p}_{\mathrm{x}}$ final states. These are virtually unchanged across a large dihedral range $\left(0^{\circ}<d_{\mathrm{CNNH}}<95^{\circ}\right)$, where the agostic interaction cannot form, yet once direct $\mathrm{C} \rightarrow \mathrm{Ni}$ orbital overlap is feasible $\left(100^{\circ}<\mathrm{d}_{\mathrm{CNNH}}<120^{\circ}\right)$, the $\mathrm{Ni} 4 \mathrm{p}_{\mathrm{x}, \mathrm{z}}$ splitting decreases as anticipated. The $\mathrm{Ni} 4 \mathrm{p}$ final states are therefore highly sensitive to the location and 
orientation of the methyl group and thus reports directly on the presence of the bis-agostic interaction. (Fig. 5b).
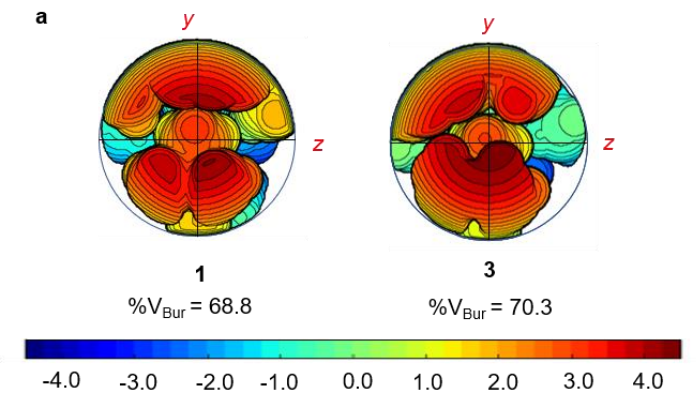

c

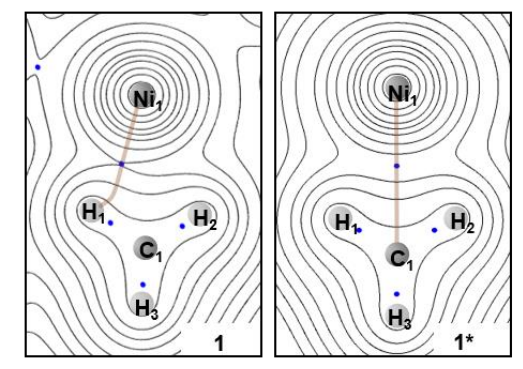

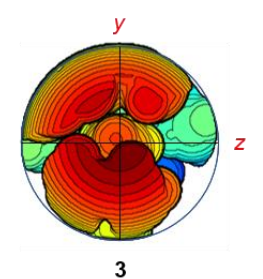

$\% \mathrm{~V}_{\text {Bur }}=70.3$

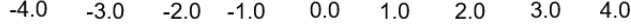

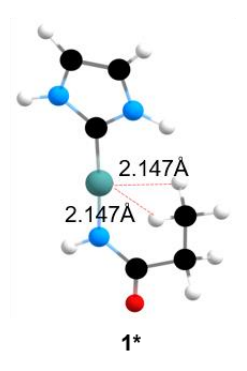

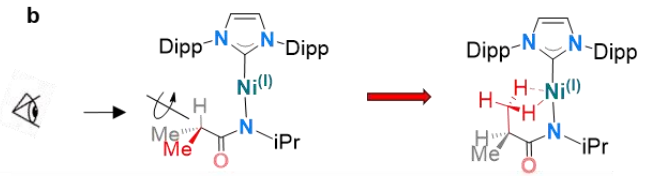

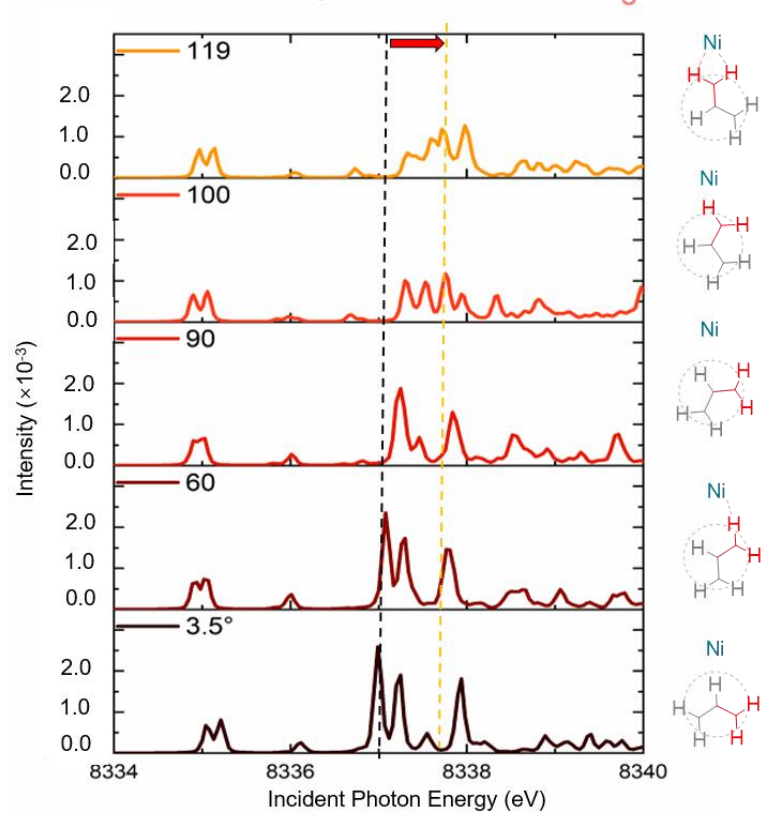

Fig. 5 | a, Topographic steric maps of agostic complexes $\mathbf{1}$ and $\mathbf{3}$. Steric maps based on the density functional theory (DFT)-optimised structures of agostic $\mathrm{Ni}(\mathrm{I})$ complexes 1, 3. The steric maps are viewed down the $x$-axis (shown in yz plane), showing the protrusion of bulk into the metal coordination sphere (defined with a radius of $4.5 \AA$ ); The isocontour scheme, in $\AA$, is shown at the bottom. The red and blue zones indicate the more- and less-hindered zones, respectively. \%VBur, percentage of buried volume. $\mathbf{b}$, Rotational relaxed scan of 2 along dihedral angle Ni-N-C-H. TD-DFT calculated Ni K-edge XAS spectra of important structures were calculated. c, AIM contour map of the electron density in the $\mathrm{H}-\mathrm{Ni}-\mathrm{H}$ plane of complex 1 and its simplified version $1^{*}$ showing bcps as blue dots and bond paths as brown lines. All possible steric substitutes in a simplified $1^{*}$ structure including 2,6-diisopropylphenyl (Dipp) from NHC ligand, methyl (Me) and isopropyl (iPr) from amide ligand were replaced with hydrogen.

As noted previously, the specific geometry of the agostic interaction is very sensitive to the steric constraints imposed by the pendant substituents of the NHC. To explore this even further, a simplified model was constructed to focus exclusively on the electronic factors controlling the formation of the $\sigma_{\mathrm{CH}} \rightarrow \mathrm{Ni}_{4 \mathrm{p}}$ agostic interaction. In this simplified model $\left(1^{\star}\right)$, electron density analysis using the Atoms In Molecules formalism (AIM) ${ }^{43}$ reveals a single bond critical point (Fig. 5c) connecting the carbon atom from the methyl group and the metal ion. The electron density value $(0.0299)$ and bond energy $(-41.2 \mathrm{~kJ} / \mathrm{mol})$ are in good agreement with a previous AIM analysis on $1 .{ }^{34}$ However, the orientation of the methyl group in the full complex is slightly twisted (at a small energy cost of $\sim 0.08 \mathrm{~kJ} / \mathrm{mol}$ ), which leads to a deviation of the agostic bcp (Fig $5 \mathrm{c} \&$ Supplementary Fig.19). In both cases, the results differ markedly from that of other bis-agostic 
complexes, where two distinct bcp are observed. ${ }^{44,45}$ The presence of a single bond critical point connecting the methyl group to the metal highlights the unique character of these electron-rich $\mathrm{Ni}(\mathrm{I})$ species, which cannot make use of $3 \mathrm{~d}$ states to accept electron density in forming the agostic bond. A second-order perturbation analysis using the natural bond orbital (NBO) formalism was also performed at a higher level of theory (B3LYP/def ${ }_{2}$ TZVPP) to explore the nature of the $\mathrm{Ni} 4 \mathrm{p}$ interactions. For $\mathbf{1}$, the analysis indicates a slight asymmetry and suggests two unsymmetrical $\sigma$ interactions involving $\sigma_{\mathrm{CH}} \rightarrow \mathrm{Ni} 3 \mathrm{~d} / 4 \mathrm{p}$ donation $\left(\left[\mathrm{E}(2)\left(\mathrm{H}_{1}\right): 103.1 \mathrm{~kJ} / \mathrm{mol}, \mathrm{E}(2)\left(\mathrm{H}_{2}\right): 115.4 \mathrm{~kJ} / \mathrm{mol}\right)\right.$, which is much stronger than typical agostic interactions (See comparisons of different theory level in Supplementary Table 22-24). ${ }^{10,13,42,46}$
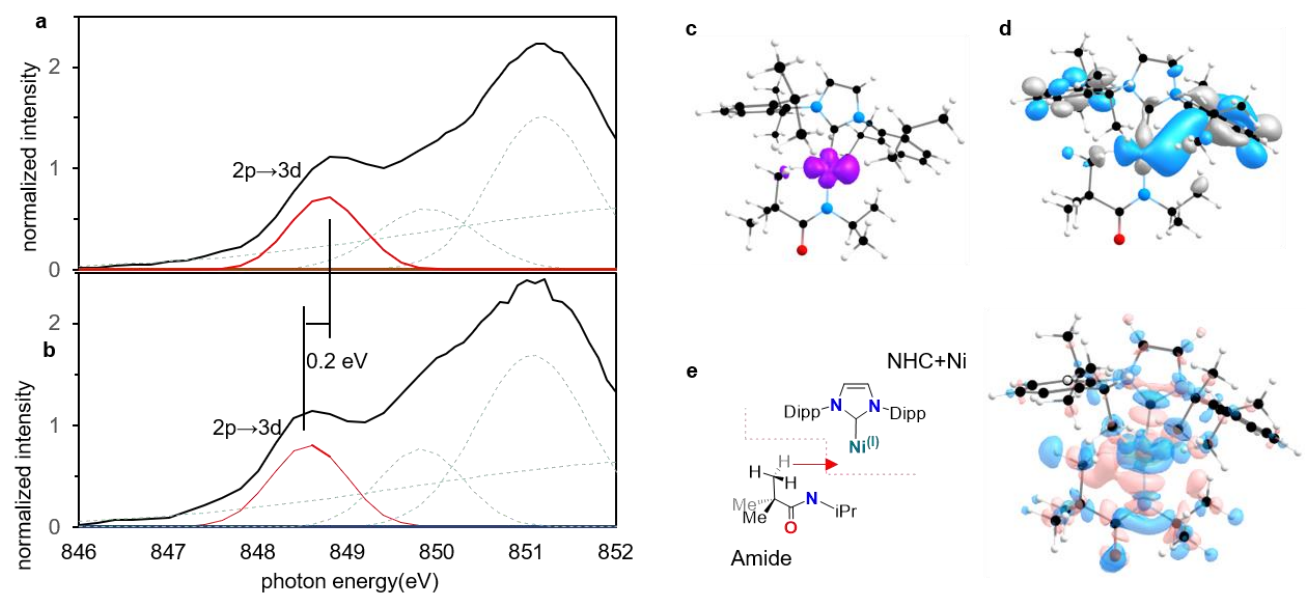

Fig. 6 | a, Normalized Ni L3-edge PFY XANES edge spectra of agostic complex $\mathbf{1}$ and $\mathbf{b}$, non-agotisc complex 2. Four gaussian equations were used to simulate the pre-edge $L_{3}$ edge feature. $\mathbf{c}$, the spin density of complex 1. d, SOMO of complex 1 contributed by $3 d_{x^{2}-y^{2}}$ orbital. e, overall charge redistribution from amide fragment to $\mathrm{NHC}-\mathrm{Ni}$ fragment (blue = decreased electron density, pink = increasing electron density).

Ni $L_{2,3}$-edge XAS spectra. To confirm that these agostic interactions are dominated by $\mathrm{Ni} 4 \mathrm{p}$ and not strongly affected by $\mathrm{Ni} 3 \mathrm{~d}$ contributions, $\mathrm{Ni} \mathrm{L}_{2,3}$-edge XAS spectra were obtained for complexes 1, 2. In these spectra, Ni 2p-3d transitions are electric dipole allowed, thus permitting a more detailed look at Ni 3d contributions.

Fits to the $\mathrm{Ni}_{3}$ edges required inclusion of four features for the spectra of both 1 and 2 (See the assignment of each Gaussian curve in Supplementary Table 18). The lowest energy feature in the spectrum correlates directly with feature A from the Ni K-edge spectra, but with significantly greater intensity. This $3 \mathrm{~d} \leftarrow 2 \mathrm{p}$ feature differs by $\sim 0.2 \mathrm{eV}$ between the two complexes (from 2848.6 $\mathrm{eV}$ to $1848.8 \mathrm{eV}$ ). TD-DFT calculations were also performed and predicted an increase of $\sim 0.3$ $\mathrm{eV}$ for this feature from 1 to 2 (Supplementary Fig 16, Supplementary Table 19 \& 20). This feature correlates with a transition to the previously identified $\mathrm{Ni} 3 \mathrm{~d}_{\times 2-\mathrm{y} 2}$-like SOMO and reflects minimal differences in the absence/presence of the agostic interaction. Although orbital interactions 
between the half-occupied $\mathrm{Ni} 3 \mathrm{~d}_{x 2-\mathrm{y} 2}$ orbital are possible with the agostic methyl group, these effects are mitigated by Pauli Repulsion (Fig 6c,d). The energy shift is consistent with a slight increase in electron density at the metal centre in the agostic complex as observed in electron density difference map (Fig. 6e). Electron donation from the methyl group into the valence Ni $4 p_{x}$ causes a slight increase in the energy of the metal valence orbitals. ${ }^{46,47}$ We note that Pauli repulsion with the agostic methyl group is also expected to raise the energy of the $3 \mathrm{~d} \leftarrow 2 p$ feature. Notably, however, the observed effects are much smaller than those observed in the $\mathrm{Ni} 4 \mathrm{p}$ valence states, thus confirming the predominance of the $\mathrm{Ni} 4 \mathrm{p}$ contributions to the strong bisagostic interaction in these complexes.

\section{Conclusion}

Herein, we report a fundamentally new form of bis-agostic interaction where the higher energy metal $4 p$ orbitals are critical in the creation of a strong carbon-metal interaction. The metal $3 d$ orbitals are essentially unavailable and thus alternative pathways are required. The specific nature of the parent linear $\mathrm{Ni}(\mathrm{I})$ complexes allows for a novel mode of bis-agostic bonding. This agostic interaction has a major impact on the x-ray spectroscopy allowing for both experimental and computational estimates of the strength of the different contributions. The bonding is dominated by the direct $\mathrm{C} 2 \mathrm{p}-\mathrm{N} 4 \mathrm{p}$ sigma donation, with minor contributions from $\sigma_{\mathrm{CH}}$ to Ni4p pi donation. This novel mode of binding is a particularly unexpected example of the importance of transition metal $p$ orbitals in defining the nature of organometallic bonding. We are now actively seeking to exploit this new binding mode to uncover unique reactivity in such systems.

\section{Methods}

\section{Synthetic Methods}

All experiments were carried out employing standard Schlenk techniques under an atmosphere of dry nitrogen employing degassed, dried solvents. Complex 1-2 used in this study are prepared according to literature procedures. Complex 3-4 are referred to Dr. D. Dawson Beattie's thesis. Celitee $₫$ was set in an oven at $180^{\circ} \mathrm{C}$ for at least 24 hours, then brought into a glovebox overnight following standard procedures. Nujol was dried over activated molecular sieves, then degassed by three freeze-pump-thaw cycles. Pyridine and acetonitrile were purchased from Sigma-Aldrich, dried over $\mathrm{CaH}_{2}$, and degassed by three freeze-pumpthaw cycles. $d_{6}$-benzene was purchased from Cambridge Isotope Laboratories Inc., dried over sodium 
metal, and degassed by three freeze-pump-thaw cycles. THF and $\mathrm{Et}_{2} \mathrm{O}$ were dried over sodium metal and degassed by three freeze-pump-thaw cycles. Hexanes and toluene were either dried over sodium metal or passed over activated alumina columns into Teflon sealed Schlenk flasks and degassed by three freezepump-thaw cycles. NMR spectra were recorded on Bruker Avance 300, 400, or $600 \mathrm{MHz}$ spectrometers. 1H NMR spectra are reported in parts per million $(\mathrm{ppm})$ and were referenced. ${ }^{48}$ to residual solvent: $1 \mathrm{H}\left(\mathrm{C}_{6} \mathrm{D}_{6}\right): \delta 7.16 ; 13 \mathrm{C}\left(\mathrm{C}_{6} \mathrm{D}_{6}\right): \delta$ 128.06; coupling constants are reported in $\mathrm{Hz}$. 13C NMR spectra were performed as proton-decoupled experiments and are reported in ppm. The multiplicities are abbreviated as follows: $s=$ singlet, $d$ = doublet, $d d=$ doublet of doublets, $t=$ triplet, sept = septet. NMR spectra are shown using MestReNova 6.0.2 NMR processing software. Integrations of paramagnetic compounds 1-4 were not possible. EI-MS data were obtained using a Kratos MS-50 spectrometer (70 eV source). Elemental analyses were recorded on a Carlo Erba EA 1108 elemental analyser. IR spectroscopy was performed using Nujol mulls between two $\mathrm{NaCl}$ salt plates on a Thermo Electron Corporation Nicolet $4700 \mathrm{FT}-\mathrm{IR}$ spectrometer.

\section{Crystallographic Methods}

All measurements of complex $\mathbf{3}$ and $\mathbf{4}$ were made on a Bruker APEX DUO diffractometer with a TRIUMPH curved-crystal monochromator with Mo-Ka radiation. The data were collected at a temperature of $-183.0 \pm 0.1^{\circ} \mathrm{C}$. Data were collected in an of $\phi$ and $\omega 0.5^{\circ}$ oscillations using 20.0 -second exposures. The crystal-to-detector distance was $40.14 \mathrm{~mm}$. Data were collected and integrated using the Bruker SAINT software package. Data were corrected for absorption effects using the multi-scan technique (SADABS). The data were corrected for Lorentz and polarisation effects. All non-hydrogen atoms were refined anisotropically. All hydrogen atoms were placed in calculated positions. The weighting scheme was based on counting statistics. Neutral atom scattering factors were taken from Cromer and Waber. Anomalous dispersion effects were included in $F_{\text {calc; }}$ the values for $f^{\prime}$ and f" were those of Creagh and McAuley. The values for the mass attenuation coefficients are those of Creagh and Hubbell. All refinements were performed using the SHELXL-2013 via the OLEX2 interface. Additional EADP (2) and SADI (1) restraints were used in the refinement process of complex 4. All restraints led to improved model statistics. More crystallographic details are given in Supplementary Material.

\section{Computational Methods}


Density Functional Theory Calculations Initial geometries for all molecules were obtained from crystallographic coordinates (where available) or constructed from standard models. Geometry optimisations and numerical frequency calculations were performed using version 4.21 of the ORCA computational chemistry package. ${ }^{49}$. Molecular geometries were optimised using the PBE0 functional ${ }^{34}$ and all-electron basis sets $\left(\text { def }_{2}-\mathrm{TZVP}\right)^{50}$ for $\mathrm{Ni}$ centre and def $_{2}$-SVP for all the rest atoms. Statistical mechanics calculations of entropic and thermal effects were performed using the rigid rotor and harmonic oscillator approximations at $298.15 \mathrm{~K}$ and 1 atm. Computational efficiency was improved by applying the RI approximation (RIJCOSX) for the hybrid functional. ${ }^{51}$ All calculations were performed with integration grid (ORCA Grid4). XAS TD-DFT (X-ray absorption Time-dependent DFT) calculations were performed with a dense integration grid (Gird6) for better implementing Scalar relativistic effects by using ZORA8 corrections and reduced by using MOanlayzer software. $\mathrm{NBO}^{52}$ calculations were calculated with Gaussian 09 program package ${ }^{13}$, AIM $^{43}$ calculations were performed in Multiwfn ${ }^{53}$ software by using NBO outputs using D3LYP39/def 2 -TZVPP level. All calculations were run on the UBC Chemistry Abacus cluster and on the Cedar of Compute Canada cluster

\section{X-ray Absorption Spectroscopy}

All the samples used in XAS $\mathrm{Ni} \mathrm{K}$ edge and $\mathrm{L}$ edge experiments were analysed as solids under anaerobic conditions and diluted in boron nitride (20-50\% by weight). Samples used in XAS Ni K-edge quickly frozen under liquid nitrogen environment. XAS Ni K-edges were acquired the SSRL beamline 73 , which is equipped with a $\mathrm{Si}(220) \phi=90^{\circ}$ double crystal monochromator, a $9 \mathrm{keV}$ cut-off mirror, and a He cryostat (at $20 \mathrm{~K}$ ). Data were collected using a Canberra 30-element Ge solid-state detector with a 3mm Co filter. Data averaging and energy calibration were performed using SixPack and the AUTOBK algorithm available in the Athena software package was employed for data reduction and normalisation.

XAS L edge spectra were recorded at beamline 10-1 at the Stanford Synchrotron Radiation Lightsource (SSRL). An array of transition-edge-sensor (TES) detectors were used to obtain high energy resolution partial fluorescence yield (PFY) data. The TES array consists of 220 operational detectors spanning a total active area of $1.9 \mathrm{~mm}^{2}$. While the monochromator was scanned across the intended energy region (e.g., 840-960 eV), the total electron yield from the samples was measured using a channeltron electron multiplier. The incident beam intensity was monitored via a gold grid and used as Io to normalise the total electron yield signal $I_{1}$. For comparison, the normalised $\left(I_{1} / l_{0}\right)$ spectra were renormalised to each other's 
maximum. The scans were recorded with a step size of $0.3 \mathrm{eV}$ and an integration time of $1 \mathrm{~s} / \mathrm{pt}$. The energy resolution of the incident radiation is $\sim 0.3 \mathrm{eV}$. Each final spectrum was the sum of five scans from different sample spots. Energy calibration was performed using $\mathrm{NiF}_{2}$, with an $\mathrm{L}_{3}$-edge feature at 852.7 eV. 54 To minimise self-absorption in the fluorescence data, the incident angle was set at $55^{\circ}$ with respect to the sample surface. All samples were measured at room temperature. To minimise radiation damage, a defocused beam (about $1 \times 1 \mathrm{~mm}^{2}$ ) was used.

\section{Data availability}

The authors declare that the main data supporting the findings of this study are available within the article and its Supplementary Information files. Extra data are available from the corresponding author upon request.

\section{References}

1. Gandeepan, P. et al. 3d Transition Metals for C-H Activation. Chemical Reviews vol. 119 2192-2452 (2019).

2. Organotransition Metal Chemistry: from Bonding to Catalysis. University Science Books https://hartwig.cchem.berkeley.edu/book/ (2010).

3. Calleja, J. et al. A steric tethering approach enables palladium-catalysed C-H activation of primary amino alcohols. Nat. Chem. 7, 1009-1016 (2015).

4. Friis, S. D., Johansson, M. J. \& Ackermann, L. Cobalt-catalysed C-H methylation for late-stage drug diversification. Nat. Chem. 12, 511-519 (2020).

5. Etienne, M. \& Weller, A. S. Intramolecular C-C agostic complexes: C-C sigma interactions by another name. Chemical Society Reviews vol. 43 242-259 (2014).

6. Bernskoetter, W. H., Schauer, C. K., Goldberg, K. I. \& Brookhart, M. Characterization of a Rhodium(I) $\sigma-M e t h a n e$ complex in solution. Science (80-. ). 326, 553-556 (2009).

7. Pike, S. D. et al. Solid-state synthesis and characterization of $\sigma$-alkane complexes, $[\mathrm{Rh}(\mathrm{L} 2)(\eta 2, \eta 2-\mathrm{C} 7 \mathrm{H} 12)][\mathrm{BArF} 4](\mathrm{L} 2$ = bidentate chelating phosphine). J. Am. Chem. Soc. 137, 820-833 (2015).

8. Brookhart, M., Green, M. L. H. \& Parkin, G. Agostic interactions in transition metal compounds. Proceedings of the National Academy of Sciences of the United States of America vol. 104 6908-6914 (2007).

9. Labinger, J. A. \& Bercaw, J. E. Understanding and exploiting C-H bond activation. Nature vol. 417 507-514 (2002).

10. Scherer, W. \& McGrady, G. S. Agostic interactions in d0 metal alkyl complexes. Angewandte Chemie - International Edition vol. 43 1782-1806 (2004).

11. Lein, M. Characterization of agostic interactions in theory and computation. Coordination Chemistry Reviews vol. 253 625-634 (2009).

12. Lin, X., Wu, W. \& Mo, Y. Agostic Interactions in Early Transition-Metal Complexes: Roles of Hyperconjugation, Dispersion, and Steric Effect. Chem. - A Eur. J. 25, 6591-6599 (2019).

13. Scherer, W. et al. The Nature of $\beta$-Agostic Bonding in Late-Transition-Metal Alkyl Complexes. Angew. Chemie Int. Ed. 50, 2845-2849 (2011).

14. Boutadla, Y., Davies, D. L., Macgregor, S. A. \& Poblador-Bahamonde, A. I. Mechanisms of C-H bond activation: Rich synergy between computation and experiment. Journal of the Chemical Society. Dalton Transactions 5820-5831 (2009) doi:10.1039/b904967c.

15. Gorelsky, S. I., Lapointe, D. \& Fagnou, K. Analysis of the concerted metalation-deprotonation mechanism in palladiumcatalyzed direct arylation across a broad range of aromatic substrates. J. Am. Chem. Soc. 130, 10848-10849 (2008). 
16. Jensen, M. P. et al. Reductive elimination/oxidative addition of carbon-hydrogen bonds at $\mathrm{Pt}(\mathrm{IV}) / \mathrm{Pt}(\mathrm{II})$ centers: Mechanistic studies of the solution thermolyses of TpMe2Pt(CH3)2H. J. Am. Chem. Soc. 125, 8614-8624 (2003).

17. Hartwig, J. F. et al. Rhodium Boryl Complexes in the Catalytic, Terminal Functionalization of Alkanes. J. Am. Chem. Soc. 127, 2538-2552 (2005).

18. Flores, J. A. et al. Methane activation and exchange by titanium-carbon multiple bonds. Chem. Sci. 2, 1457 (2011).

19. Cavaliere, V. N. et al. Room Temperature Dehydrogenation of Ethane to Ethylene. J. Am. Chem. Soc. 133, 10700-10703 (2011).

20. Waltz, K. M. \& Hartwig, J. F. Selective functionalization of alkanes by transition-metal boryl complexes. Science (80-. ). 277, 211-213 (1997).

21. Waltz, K. M., He, X., Muhoro, C. \& Hartwig, J. F. Hydrocarbon Functionalization by Transition Metal Boryls. J. Am. Chem. Soc. 117, 11357-11358 (1995).

22. Chen, H., Schlecht, S., Semple, T. C. \& Hartwig, J. F. Thermal, catalytic, regiospecific functionalization of alkanes. Science (80-. ). 287, 1995-1997 (2000).

23. Hartwig, J. F., Bhandari, S. \& Rabien, P. R. Addition of Catecholborane to a Ruthenium-Alkyl: Evidence for $\sigma$ Bond Metathesis with a Low-Valent, Late Transition Metal. J. Am. Chem. Soc. 116, 1839-1844 (1994).

24. Brammer, L. et al. Hydrogen bonding by cisplatin derivative: Evidence for the formation of $\mathrm{N}-\mathrm{H} \cdots \mathrm{Cl}$ and $\mathrm{N}-\mathrm{H} \cdots \mathrm{Pt}$ bonds in [NPrn4] 2\{[PtCl4]-cis-[PtCl2(NH 2Me)2]\}. J. Chem. Soc. Chem. Commun. 443-445 (1987) doi:10.1039/C39870000443.

25. Scherer, W. et al. Anagostic Interactions under Pressure: Attractive or Repulsive? Angew. Chemie Int. Ed. 54, 2505-2509 (2015).

26. Huynh, H. V., Wong, L. R. \& Ng, P. S. Anagostic interactions and catalytic activities of sterically bulky benzannulated N-heterocyclic carbene complexes of nickel(II). Organometallics 27, 2231-2237 (2008).

27. Kandiah, M., McGrady, G. S., Decken, A. \& Sirsch, P. [(Triphos)Ni(ๆ2-BH4)]: An unusual nickel(I) borohydride complex. Inorg. Chem. 44, 8650-8652 (2005).

28. Gloaguen, Y. et al. Phosphinoborane and Sulfidoborohydride as Chelating Ligands in Polyhydride Ruthenium Complexes: Agostic $\sigma$-Borane versus Dihydroborate Coordination. Angew. Chemie Int. Ed. 48, 2964-2968 (2009).

29. Beck, R. \& Johnson, S. A. Structural similarities in dinuclear, tetranuclear, and pentanuclear nickel silyl and silylene complexes obtained via Si-H and Si-C activation. Organometallics 31, 3599-3609 (2012).

30. Baratta, W. et al. Nonclassical vs Classical Metal $\cdots H 3 C-C$ Interactions: Accurate Characterization of a 14-Electron Ruthenium(II) System by Neutron Diffraction, Database Analysis, Solution Dynamics, and DFT Studies. J. Am. Chem. Soc. 126, 5549-5562 (2004).

31. Maria, L. et al. Very small and soft scorpionates: Water stable technetium tricarbonyl complexes combining a bis-agostic (k3-H, H, S) binding motif with pendant and integrated bioactive molecules. J. Am. Chem. Soc. 128, 14590-14598 (2006).

32. Crosby, S. H., Clarkson, G. J. \& Rourke, J. P. A delicate balance between sp2 and sp3 C-H bond activation: A Pt(II) complex with a dual agostic interaction. J. Am. Chem. Soc. 131, 14142-14143 (2009).

33. Riddlestone, I. M. et al. Isolation of [Ru(IPr) $2(\mathrm{CO}) \mathrm{H}]+(\mathrm{IPr}=1,3$-Bis(2,6-diisopropylphenyl)imidazol-2-ylidene) and Reactivity toward E-H (E = H, B) Bondsboranes H $3 \mathrm{~B} \cdot \mathrm{NR} 2 \mathrm{H}(\mathrm{R}=\mathrm{Me}, \mathrm{H})$ gave [Ru(IPr) 2 (CO) $3 \mathrm{H}] \mathrm{BAr} 4 \mathrm{~F}(3)$, $[\mathrm{Ru}(\mathrm{IPr}) 2(\mathrm{CO})(\eta$ 2-H 2$) \mathrm{H}] \mathrm{BAr} 4 \mathrm{~F}(4)$, and $[\mathrm{Ru}(\mathrm{IPr}) 2(\mathrm{CO})(\kappa 2-\mathrm{H} 2 \mathrm{BH} \cdot \mathrm{NR} 2 \mathrm{H}) \mathrm{H}] \mathrm{BAr} 4 \mathrm{~F}$ Chart 1. Organometallics 35, 1301-1312 (2016).

34. Beattie, D. D., Bowes, E. G., Drover, M. W., Love, J. A. \& Schafer, L. L. Oxidation State Dependent Coordination Modes: Accessing an Amidate-Supported Nickel(I) $\delta$-bis $(\mathrm{C}-\mathrm{H})$ Agostic Complex. Angew. Chemie - Int. Ed. 55, 13290-13295 (2016).

35. Beattie, D. D., Lascoumettes, G., Kennepohl, P., Love, J. A. \& Schafer, L. L. Disproportionation Reactions of an Organometallic Ni(I) Amidate Complex: Scope and Mechanistic Investigations. Organometallics 37, 1392-1399 (2018).

36. Furenlid, L. R., Renner, M. W. \& Fujita, E. XAS studies of Ni(I), Ni(II), and Ni(III) complexes. Phys. B Phys. Condens. Matter 208-209, 739-742 (1995).

37. Uyeda, C. \& Peters, J. C. Access to formally Ni(i) states in a heterobimetallic NiZn system. Chem. Sci. 4, 157-163 (2013).

38. Schuth, N. et al. Biomimetic mono- and dinuclear Ni(I) and Ni(II) complexes studied by X-ray absorption and emission spectroscopy and quantum chemical calculations. J. Phys. Conf. Ser. 712, 012134 (2016).

39. Tomson, N. C. et al. Re-evaluating the $\mathrm{Cu} \mathrm{K}$ pre-edge XAS transition in complexes with covalent metal-ligand interactions. Chem. Sci. 6, 2474-2487 (2015).

40. Kau, L. shan, Spira-solomon, D. J., Spira-solomon-penner-hahn, J. E., Hodgson, K. O. \& Solomon, E. I. X-ray Absorption 
Edge Determination of the Oxidation State and Coordination Number of Copper: Application to the Type 3 Site in Rhus vernicifera Laccase and Its Reaction with Oxygen. J. Am. Chem. Soc. 109, 6433-6442 (1987).

41. Titus, C. J. et al. L-edge spectroscopy of dilute, radiation-sensitive systems using a transition-edge-sensor array. J. Chem. Phys. 147, 214201 (2017).

42. Von Frantzius, G., Streubel, R., Brandhorst, K. \& Grunenberg, J. How strong is an agostic bond? Direct assessment of agostic interactions using the generalized compliance matrix. Organometallics 25, 118-121 (2006).

43. Bader, R. F. W. A Quantum Theory of Molecular Structure and Its Applications. Chem. Rev. 91, $893-928$ (1991).

44. Riddlestone, I. M. et al. Isolation of $[\mathrm{Ru}(\mathrm{IPr}) 2(\mathrm{CO}) \mathrm{H}]+(\operatorname{IPr}=1,3-\mathrm{Bis}(2,6$-diisopropylphenyl)imidazol-2-ylidene $)$ and Reactivity toward E-H (E = H, B) Bonds. Organometallics 35, 1301-1312 (2016).

45. Saha, K. et al. Design, Synthesis, and Chemistry of Bis( $\sigma)$ borate and Agostic Complexes of Group 7 Metals. Chem. - A Eur. J. 23, 9812-9820 (2017).

46. He, W. \& Kennepohl, P. Direct experimental evaluation of ligand-induced backbonding in nickel metallacyclic complexes. Faraday Discuss. 220, 133-143 (2019).

47. Desnoyer, A. et al. The importance of ligand-induced backdonation in the stabilization of square planar d10 Nickel $\pi$ complexes. Chem. - A Eur. J. (2019) doi:10.1002/chem.201805987.

48. Fulmer, G. R. et al. NMR chemical shifts of trace impurities: Common laboratory solvents, organics, and gases in deuterated solvents relevant to the organometallic chemist. Organometallics 29, 2176-2179 (2010).

49. Neese, F., Wennmohs, F., Hansen, A. \& Becker, U. Efficient, approximate and parallel Hartree-Fock and hybrid DFT calculations. A 'chain-of-spheres' algorithm for the Hartree-Fock exchange. Chem. Phys. 356, 98-109 (2009).

50. Schäfer, A., Huber, C. \& Ahlrichs, R. Fully optimized contracted Gaussian basis sets of triple zeta valence quality for atoms Li to Kr. J. Chem. Phys. 100, 5829-5835 (1994).

51. Marenich, A. V., Cramer, C. J. \& Truhlar, D. G. Universal solvation model based on solute electron density and on a continuum model of the solvent defined by the bulk dielectric constant and atomic surface tensions. J. Phys. Chem. B 113, 6378-6396 (2009).

52. Glendening, E. D., Landis, C. R. \& Weinhold, F. NBO 6.0: Natural bond orbital analysis program. J. Comput. Chem. 34, 1429-1437 (2013).

53. Lu, T. \& Chen, F. Multiwfn: A multifunctional wavefunction analyzer. J. Comput. Chem. 33, 580-592 (2012).

54. Olalde-Velasco, P., Jiménez-Mier, J., Denlinger, J. \& Yang, W.-L. Atomic multiplets at the L 2 , 3 edge of 3 d transition metals and the ligand Kedge in X-ray absorption spectroscopy of ionic systems. Phys. Rev. B 87, 245136 (2013).

Acknowledgement. The authors thank The University of British Columbia (4YF to D.D.B., Laird to D.D.B., W. H. E.G.B.), the government of Canada (Vanier Canada Graduate Scholarship to E.G.B.), and the Globalink Graduate Fellowship from Mitacs Canada to W.H. NSERC (Discovery Grant to P.K). NSERC (Discovery, CREATE, and Instrumentation Grants to J.A.L. and L.L.S.), This work was undertaken, in part, thanks to funding from the Canada Research Chairs program (L.L.S.). Use of the Stanford Synchrotron Radiation Lightsource, SLAC National Accelerator Laboratory, is supported by the U.S. Department of Energy, Office of Science, Office of Basic Energy Sciences under Contract No. DE-AC02-76SF00515. The SSRL Structural Molecular Biology Program is supported by the DOE Office of Biological and Environmental Research, and by the National Institutes of Health, National Institute of General Medical Sciences 
(including P41GM103393). The contents of this publication are solely the responsibility of the authors and do not necessarily represent the official views of NIGMS or NIH. Thanks to the helpful discussion with Kris Altus on $\mathrm{C}-\mathrm{H}$ activation.

Author contributions. WH and PK conceived the study. PK, JAL, LLS supervised the study. WH performed the spectroscopic and DFT computational studies. DDB performed the synthesis and collected X-ray diffraction data. EB helped perform the NBO calculation and provided computational suggestions. HZ performed the EXAFS analysis. PK and WH wrote the paper. All authors discussed the results and participated in revising and finalizing the manuscript.

Additional Information. Supplementary information and chemical compound information are available in the online version of the paper. Reprints and permissions information is available online at www.nature.com/reprints. Correspondence and requests for materials should be addressed to P.K.

Competing Interests. The authors declare no competing interests.

Table 1. | Four Lorentzian curves were implemented to simulate the experimental Ni K-edge spectra for comparing the transition energy in TD-DFT calculated Ni K-edge XAS spectra of complexes 1-4. Most intense calculated transition energy in each feature was provided. The contribution of Nickel $3 d / 4 p$ character was normalized based on the overall $\mathrm{Ni}$ character in the $\mathrm{MO}$ acceptors contributed to each feature.

\begin{tabular}{|c|c|c|c|c|c|c|c|c|}
\hline \multirow{3}{*}{$\begin{array}{c}\text { Code } \\
\text { Transition } \\
\text { Assignment }\end{array}$} & \multicolumn{4}{|c|}{1} & \multicolumn{4}{|c|}{2} \\
\hline & Exp & \multicolumn{3}{|c|}{ TD-DFT } & \multirow{2}{*}{$\begin{array}{c}\text { Exp } \\
\text { Energy }\end{array}$} & \multicolumn{3}{|c|}{ TD-DFT } \\
\hline & Energy & Energy & $\mathrm{Ni} 3 d$ & $\mathrm{Ni} 4 p$ & & Energy & $\mathrm{Ni} 3 d$ & $\mathrm{Ni} 4 \mathrm{p}$ \\
\hline A & 8331.04 & 8330.48 & $91.45 \%$ & $8.55 \%$ & 8323.11 & 8330.19 & $90.2 \%$ & $9.4 \%$ \\
\hline B & 8335.13 & 8335.27 & $35.12 \%$ & $64.06 \%$ & 8335.20 & 8335.34 & $34.8 \%$ & $64.8 \%$ \\
\hline C & 8337.34 & 8337.84 & $7.34 \%$ & $89.84 \%$ & 8337.10 & 8337.12 & $19.0 \%$ & $75.4 \%$ \\
\hline $\mathrm{D}$ & 8337.72 & 8338.11 & $11.33 \%$ & $83.83 \%$ & 8337.97 & 8338.07 & $5.7 \%$ & $91.5 \%$ \\
\hline$\Delta \mathrm{E}(\mathrm{C} / \mathrm{D})$ & 0.38 & 0.27 & & & 0.87 & 0.95 & & \\
\hline Code & \multicolumn{4}{|c|}{3} & \multicolumn{4}{|c|}{4} \\
\hline Transition & Exp & \multicolumn{2}{|c|}{ TD-DFT } & & Exp & \multicolumn{2}{|c|}{ TD-DFT } & \\
\hline Assignment & Energy & Energy & $\mathrm{Ni} 3 d$ & $\mathrm{Ni} 4 p$ & Energy & Energy & $\mathrm{Ni} 3 d$ & $\mathrm{Ni} 4 p$ \\
\hline A & 8331.57 & 8330.43 & $77.5 \%$ & $7.1 \%$ & 8327.81 & 8330.13 & $78.1 \%$ & $0.00 \%$ \\
\hline B & 8335.27 & 8335.21 & $48.7 \%$ & $47.2 \%$ & 8335.72 & 8335.25 & $34.9 \%$ & $64.7 \%$ \\
\hline C & 8337.32 & 8337.38 & $11.9 \%$ & $78.5 \%$ & 8336.90 & 8337.1 & $5.9 \%$ & $92.8 \%$ \\
\hline
\end{tabular}




\begin{tabular}{c|cccccccc}
$\mathrm{D}$ & 8337.78 & 8337.92 & $5.9 \%$ & $85.5 \%$ & 8338.10 & 8338.07 & $1.2 \%$ & $97.8 \%$ \\
$\Delta \mathrm{E}(\mathrm{C} / \mathrm{D})$ & 0.46 & 0.54 & & & 1.20 & 0.97 & \\
\hline
\end{tabular}

